\title{
Caustics in dark matter haloes
}

\author{
Roya Mohayaee* and Sergei F. Shandarin $^{\dagger}$ \\ *Institut d'Astrophysique de Paris, 98bis boulevard Arago, 75014 Paris, France \\ ${ }^{\dagger}$ Department of Physics and Astronomy, University of Kansas, KS 66045, U.S.A. \\ emails: roya@iap.fr ; sergei@ku.edu
}

20 February 2019

\begin{abstract}
Cold dark matter haloes are populated by high-density structures with sharplypeaked profiles known as caustics which have not yet been resolved by 3dimensional numerical simulations. Here, we derive semi-analytic expressions for the density profiles near caustics in haloes which form by self-similar accretions of dark matter with infinitesimal velocity dispersion. A simple rescaling shows that these profiles are universal: they are valid for all caustics and irrespective of physical parameters of the halo. We derive the maximum density of the caustics and show that it depends on the velocity dispersion and the caustic location. Finally, we demonstrate that there can be a significant contribution to the emission measure from dark matter particle annihilation in the caustics.
\end{abstract}

Key words: dark matter, haloes of galaxies, caustics, velocity dispersion, dark matter detection

\section{INTRODUCTION}

Dark matter particles, if collisionless and cold, would focus under gravitational instability into caustics which are formally 2D manifolds of infinite density. In three-dimensional space, caustics are determined by tangent hyperplanes $\mathbf{r}=$ const to the phase surface in the six-dimentional phase space $(\mathbf{r}, \mathbf{v})$ and bound the regions of multi-stream flow where velocity has multiple values. Once formed, caustics of a given phase volume would not disappear or overlap; a requirement of the Liouville theorem. However they can interact and merge with caustics of a different phase volume and consequently the nature of their singularity could change and they could undergo generic metamorphoses. In the case of potential flows, e.g. of light rays or cold dark matter on large scales, the singularities of the caustics and their metamorphosis have been classified up to three spatial dimensions (Arnold 1986, 1990). This classification remains intact in the presence of external or internal forces (e.g. in a self-gravitating system) for as long as the force is potential and smooth. The first and most common caustic has a density with an inverse square-root singularity (as occurs in the Zel'dovich approximation). This singularity has been proven to persist in the case of a one-dimensional Vlassov-Poisson system (Roytvarf 1994) and is the only singularity that is of relevance to the present work.

In cosmology, the study of formation and evolution of dark matter caustics has been historically and scientifically twofold. On large scales, pioneering works by Zel'dovich and his collaborators (Zel'dovich 1970, Arnold, Shandarin and Zel'dovich 1982, Shandarin and Zel'dovich 1989) showed that in a suitably-defined time and space coordinates, elements of a collisionless and self-gravitating fluid move on inertial trajectories: i.e. with their initial velocities. Thus, as in ray optics, when the path of the free-moving particles cross, density diverges, velocity becomes multivalued and caustics form. Caustics can bound regions with the morphology of filaments, sheets or clumps together forming a supercluster-void network which is remarkably similar to the mass distribution in the Universe on large scales (above $4-5 \mathrm{Mpc}$ ) as shown in numerical simulations (e.g. Sahni and Shandarin 1996) and redshift galaxy catalogs (e.g. Bharadwaj et al. 2000). However, at small scales, within collapse structures, inertial approximation of Zel'dovich breaks down.

The question arises whether caustics are relevant to the physics of dark matter at small scales, for example at the scale of a dark matter halo. Dark matter haloes can form for instance from the triaxial collapse of spherical perturbations or accretion of matter into the overdensities at the junctions of the filaments. In a cold dark matter 
Universe (with or without cosmological constant), they grow in a hierarchical manner by merging with other haloes and by accreting mass and are hosts to the formation of galaxies. In numerical simulations, they are bound overdense regions which are identified by various percolation algorithms. High resolution simulations, surprisingly, have found that the spherically-averaged equilibrium density profiles of cold dark matter haloes can be described by a power law (Dubinski \& Carlberg 1991) and universal two-parameter function (Navarro, Frenk \& White 1996, 1997). Although simulations have clearly established that many of the supposedly "relaxed" haloes still contain a large number of smaller subhaloes (Klypin et al. 1999, Moore et al. 1999), they have so far not achieved enough resolution to observe the small-scale caustics in three dimensions and they also probably suffer from spurious collisional effects (Melott et al. 1997, Splinter et al. 1998, Binney 2004) which could wash away the caustics.

Recent high resolution N-body simulations of the neutralino-dominated Universe have shown that a considerable number of the smallest haloes with masses as small as $10^{-6} M_{\odot}$ survive until the present epoch (Diemand, Moore \& Stadel 2005). These simulations suggest that there must be about $10^{15}$ such haloes in our galaxy. These haloes are expected to have very smooth caustics because there had been no smaller scale fluctuations in the initial spectrum. The results of our work are most directly applicable to this type of structures.

Analytic evaluation of the halo density profile, and the prediction of the existence of caustics inside these structures, started with the works of Gott (1975) and Gunn (1977). With the main objective of explaining the flattening of the rotation curves of the galaxies, they considered the formation of a dark matter halo from the secondary infall of matter onto an already formed galaxy (or in later works onto a spherical overdense region). In an Einstein-de Sitter Universe a spherical overdensity expands and then turns around to collapse. After collapse and at late times, the fluid motion becomes selfsimilar: its form remains unchanged when length are re-scaled in terms of the radius of the shell which is currently turning around and falling onto the galaxy. Physically selfsimilarity arises because gravity is scale-free and because mass shells outside the initial overdensity are also bound and turn around at successively later times. Self-similar solutions give power-law density profiles whose exact scaling properties depend on the central boundary conditions and on whether the fluid is collisionless or collisional (Fillmore and Goldreich 1984, Bertschinger 1985a, 1985b). The density profile obeys a power-law on the scale of the halo which provides an explanation of the flattening of the rotation curves of the galaxies. However, on smaller scales the density profile contains many spikes (i.e. caustics) of infinite density (with an artificial cutoff due to finite numerical resolution). The position and the time of formation of these caustics are among many properties which have been studied in the secondary infall model (Bertschinger 1985b). ${ }^{1}$

Such aforementioned studies not only have proven valuable for the prediction and description of large-scale structure, of dynamics and distribution of mass inside dark matter haloes and of galaxy formation but also recently for the detection of dark matter particles. Due to their significantly high density over their often already dense background, and their large number density, caustics are clearly of importance for dark matter search experiments.

In the past few years, major experiments have gone underway for the direct and indirect detection of dark matter particles. Direct detection experiments, such as DAMA and EDELWEISS, often use the annual modulation of the signal due to the orbital motion of the Earth around the Sun. Since the flux of dark matter in direct searches depends linearly on the local dark matter density, the search strategy and data analysis strongly depend on the spatial distribution of dark matter and its dynamics in the galactic halo.

The indirect-detection experiments, such as ANTARES, HESS and GLAST, search for products of annihilation of dark matter candidates (e.g. neutralinos) such as energetic neutrinos and $\gamma$-rays. In the indirect searches, the flux of the annihilation products depends quadratically on the local dark matter density. Thus the degree of clumpiness, the density profile of a dark matter halo, the presence or absence of a central supermassive black hole and finally the presence of caustics, could influence the annihilation rate and boost the $\gamma$-ray flux significantly. It has been shown that this boost is significant if there is a cusp at the centre of the halo (Stoehr 2003, Salati 2004). The accretion of dark matter into a central black hole if present in the halo, could also boost the gamma-ray flux by few orders of magnitude but again only if the dark matter profile develops a cusp at the centre (Gondolo and Silk 1999). Thus, a central core profile would not in general lead to a significant boost of the flux. However, although dark-matter-only simulations seem to show a cuspy profile in the centre of the haloes, some of the observations seem to contradict these predictions (e.g. see McGaugh et al. 2003). In addition, whether the cusp observed in the dark-matter-only simulations would survive in the presence of gas or would become less steep or else disappear due to the reaction from the baryonic gas, or is simply a numerical artifact (Binney 2004) is unclear. Caustics, on the other hand, would be inevitably present, as a direct consequence of Jeans-Vlassov-Poisson equation (e.g. see Alard \& Colombi 2004 for a recent numerical method in 1 dimension). Therefore, it is worthwhile to study the density enhancement in the

1 Although various elaborations have since been made on the secondary infall, in order to accommodate for the bi-scaling of the haloes density profile observed in simulations (e.g. see Henriksen 2004) here we concentrate on the original secondary infall model which yields a pure power law density profile. 
caustics and its possible implications for dark matter search experiments. Some previous works (Sikivie, Tkachev and Wang 1997, Bergström et al. 2001) have considered caustic formations for very cold (mainly axions with zero velocity dispersion) dark matter. In a few of these works an artificial upper cut-off for the densities of the caustics have been used (Bersgtröm et al. 2001) and in some others the non-vanishing angular momentum would stop the divergence of the densities (Sikivie, Tkachev and Wang 1997).

Although, mathematically, caustics are defined as objects with infinite density, in real physical systems their density is not infinite mainly due to the finite velocity dispersion of the fluid particles. The presence of a small velocity dispersion, e.g for neutralinos which are presently the most plausible dark matter candidates, smoothes the matter density at the caustic and gives it a finite maximum value. The question specifically addressed here is the value of this quantity and its implications for dark matter search experiences.

In view of the fact that almost all dark matter candidates have non-negligible velocity dispersion, we consider the secondary infall of dark matter with a very small but finite velocity dispersion. In this case the density profile at the very vicinity of the caustic would be affected and in addition caustics would have a physical cut-off to their density. Here, we evaluate analytic expressions for the density profile at the vicinity of the caustics and also determine the maximum density at the caustic positions. The analytic expression for the density profile of the caustics is given as a function of the initial velocity dispersion. The $\gamma$-ray emission measure from the annihilation of the neutralinos in the caustics is then evaluated. Using our results, we evaluate the position, the thickness, the density and the $\gamma$-ray emission measure for the first caustic of M31.

This article is organised as follows. In Section 2, we review the basics of the secondary infall model. In section 3 , we derive analytic expressions for the density profiles near the caustics in the presence of small velocity dispersion. In Section 4, we use our density profile and evaluate a general analytic expression for the emission measure from a typical caustic. In Section 5 we evaluate the $\gamma$-ray emission measure from dark matter annihilation in the first caustic (nearest to us) of M31. In Section 6 we conclude our main results.

\section{SELF-SIMILAR MODEL}

We consider a spherical overdensity of collisionless fluid in an Einstein-de Sitter Universe which eventually ceases expansion and turns around to collapse. The trajectory of a fluid element in radial motion obeys Newton's law

$\frac{d^{2} r}{d t^{2}}=-\frac{G m(r, t)}{r^{2}}$

where the mass, $m(r, t)$ inside a radius $r$, is not constant due to shell-crossing. At first the only way to tackle this problem seems to be via an N-body simulation. However, a major simplification arises once it is realised that the problem has a similarity solution [(Filmore \& Goldreich 1984) and (Bertschinger 1985b) and we use the notations of (Bertschinger 1985b) throughout]. The turn around radius $r_{\mathrm{ta}}(t)$ which is the only length scale in the problem is used to introduce the non-dimensional variables

$\lambda=\frac{r(t)}{r_{\mathrm{ta}}(t)} \quad ; \quad \xi=\ln \left(\frac{t}{t_{\mathrm{ta}}}\right) \quad ; \quad M(\lambda)=\frac{3}{4 \pi} \frac{m(r, t)}{\rho_{H} r_{\mathrm{ta}}^{3}}$,

where

$r_{\text {ta }}(t)=r_{\text {ita }}\left(\frac{t}{t_{\text {ita }}}\right)^{8 / 9}$

$r_{\text {ita }}$ is the initial turnaround radius, $t_{\text {ita }}$ is the initial turnaround time, $\rho_{H}$ is the Einstein-de Sitter density $\left(\rho_{H}=\right.$ $\left.1 / 6 \pi G t^{2}\right)$ and $t_{\mathrm{ta}}$ is the turnaround time for a given particle (i.e. when the particle is at its largest radius). In terms of the nondimensional variables (2) Newton's equation (1) becomes

$\frac{d^{2} \lambda}{d \xi^{2}}+\frac{7}{9} \frac{d \lambda}{d \xi}-\frac{8}{81} \lambda=-\frac{2}{9 \lambda^{2}} M(\lambda)$

which has no explicit dependence on non-dimensional time $\xi$. The equation should be solved with the initial condition (at $\xi=0$ corresponding to $t=t_{\mathrm{ta}}$ )

$\lambda=1 ; \quad \frac{d \lambda}{d \xi}=-\frac{8}{9}$,

and a prior knowledge of the mass $M(\lambda)$. In the case of the Hubble flow, there is a simple solution to equation (4), before shell-crossing, which is given by $M(\lambda)=\lambda^{3}=M_{\mathrm{ta}} e^{-2 \xi / 3}$. However, after shell-crossing occurs there are many particles having the same value of $\lambda$. This can be taken into account simply by the summation

$M(\lambda)=M_{\mathrm{ta}} \sum_{i}(-1)^{i-1} e^{-2 \xi_{i} / 3}$ 
which adds (for $i$ odd) the mass of the particles interior to $\lambda$ and subtracts (for even $i$ ) the mass exterior to $\lambda$, accounting correctly for shell-crossing. Equations (4) and (6) can be solved numerically by iteration (see Appendix A for more detailed account). Here, we take a simpler approach. At small values of $\lambda(\lambda \ll 1)$, mass becomes a power-law $M(\lambda) \approx 11.2 \lambda^{3 / 4}$ (Bertschinger 1985b). We take this fact into account and instead of solving (4) and (6) iteratively, use a simple approximation for $M(\lambda)$

$M(\lambda) \approx \frac{11.2 \lambda^{3 / 4}}{1+\lambda^{3 / 4}}$

and then solve (4) numerically at the given gravitational potential generated by the mass distribution (7).

As shown in Appendix A by Figure A1 the approximation (7) generates relatively small errors. A notable discrepancy between the approximation (7) and expression (6) appears only at the relatively large values of $\lambda \sim 1$. However, for all the caustics under consideration the value of $\lambda$ is far less than one [the largest value of $\lambda$ for the first caustic is at $\lambda \approx 0.36$ (see the table in Fig. A1)].

Solutions to equations (4) and (6), give a power-law density profile convolved with many sharp spikes (the caustics). As a particle expands to its turnaround radius, it collapses and re-expands again to its new maximum radius, which gives the time and position of the first caustics. It then re-collapses and re-expands to the position of the second caustic and so on (see Fig. A2). The calculation of the halo density profile itself is not the subject of this work. Here we are primarily concerned with the calculation of the density profile near the caustics and the maximum density at the caustics in the case of dark matter with finite velocity dispersion.

\section{MAXIMUM DENSITY IN COSMOLOGICAL CAUSTICS}

\subsection{Cold medium}

First, we derive the equations of motion in terms of physical time $t$ and radius $r$. From the definitions (2) one can easily obtain

$\begin{aligned} t & =t_{\text {ita }}\left(\frac{R_{\mathrm{ta}}}{r_{\text {ita }}}\right)^{9 / 8} e^{\xi}, \\ r & =R_{\mathrm{ta}} \exp \left(\frac{8}{9} \xi\right) \lambda(\xi),\end{aligned}$

where

$R_{\mathrm{ta}} \equiv r_{\mathrm{ta}}\left(t_{\mathrm{ta}}\right)=r_{\mathrm{ita}}\left(\frac{t_{\mathrm{ta}}}{t_{\mathrm{ita}}}\right)^{8 / 9}$

is the turnaround radius reached by a particle at its turnaround time (which is equivalent to $r_{\mathrm{ta}}^{\prime}$ in Bertschinger 1985b). Solving equation (8) for $\xi$ one can obtain in terms of the physical coordinates and function $\lambda=\lambda(\xi)$ the explicit solution

$r\left(R_{\mathrm{ta}}, t\right)=r_{\mathrm{ita}}\left(\frac{t}{t_{\mathrm{ita}}}\right)^{8 / 9} \lambda\left\{\ln \left[\frac{t}{t_{\mathrm{ita}}}\left(\frac{R_{\mathrm{ta}}}{r_{\mathrm{ita}}}\right)^{-9 / 8}\right]\right\}$.

Introducing dimensionless time $\tau$ and dimensionless coordinates $x$ and $q$

$\tau=\frac{t}{t_{\mathrm{ita}}}, \quad x=\frac{r}{r_{\mathrm{ita}}}, \quad$ and $\quad q=\frac{R_{\mathrm{ta}}}{r_{\mathrm{ita}}}$

one can further simplify equation (11)

$x=\tau^{8 / 9} \lambda\left[\ln \left(\tau q^{-9 / 8}\right)\right]$.

Equations (11) and (13) represent the mapping from Lagrangian space to Eulerian space parameterised by time.

The density can be obtained from the conservation of mass

$\rho(x)=\rho(q) \frac{q^{2}}{x^{2}}\left|\frac{d x}{d q}\right|^{-1}$,

where the ratio $d x / d q$ must be taken at the time of formation of the caustic, $\tau_{k}$. The condition of the caustic formation $d x / d q=0$ requires

$\lambda^{\prime}\left(\xi_{k}\right)=0$

where $\lambda^{\prime}=d \lambda / d \xi$ and $\xi_{k}=\tau_{k} q^{-9 / 8}$ denotes the position of a maximum of the function $\lambda(\xi)$. The derivative $d x / d q$ at the Lagrangian distance $\Delta q$ from the caustic is 
$\frac{d x}{d q}=\left(\frac{\partial^{2} x}{\partial q^{2}}\right)_{\tau_{k}} \Delta q=\frac{81}{64} \frac{\tau_{k}^{8 / 9}}{q^{2}} \lambda_{k}^{\prime \prime} \Delta q$

where $\lambda_{k}^{\prime \prime}=\lambda^{\prime \prime}\left(\xi_{k}\right)$ The relation between $\Delta x$ and $\Delta q$ can be easily found by expanding $x(\tau, q)$ [equation (13)] into the Taylor series at the time $\tau_{k}$ and using the condition of $\lambda=\max$ [given by equation (15)]

$\Delta x=\left(\frac{\partial x}{\partial q}\right)_{\tau_{k}} \Delta q+\frac{1}{2}\left(\frac{\partial^{2} x}{\partial q^{2}}\right)_{\tau_{k}} \Delta q^{2}=\frac{1}{2} \frac{81}{64} \frac{\tau_{k}^{8 / 9}}{q^{2}} \lambda_{k}^{\prime \prime} \Delta q^{2}$.

Thus, the inverse derivative $(d x / d q)^{-1}$ becomes

$\left|\frac{d x}{d q}\right|^{-1}=\frac{1}{2} \frac{8}{9} \frac{q}{\tau_{k}^{4 / 9}}\left(-\frac{\lambda_{k}^{\prime \prime}}{2}\right)^{-1 / 2}(-\Delta x)^{-1 / 2}$,

where the signs in the above equation reflect the signs of $\lambda_{k}^{\prime \prime}<0$ and $\Delta x<0$ in the vicinity of the caustic. Substituting the derivative $(d x / d q)^{-1}$, obtained above, into equation (14) one derives the density in the vicinity of a caustic (additional factor 2 must be added due to two stream flow at $\Delta x<0$ )

$\rho(\Delta x)=A_{k}(-\Delta x)^{-1 / 2}$

with

$A_{k}=\frac{2}{9} \rho_{H} \frac{M_{t a}}{\lambda_{k}^{2}} \exp \left(-\frac{2}{3} \xi_{k}\right)\left(-\frac{\lambda_{k}^{\prime \prime}}{2}\right)^{-1 / 2} x_{t a}^{1 / 2}$,

where $\rho_{H}=1 / 6 \pi G t^{2}$ is the mean density of the universe, $M_{t a}=(3 \pi / 8)^{2}, \lambda_{k}=\lambda\left(\xi_{k}\right)$, and $x_{t a}=r_{t a}(t) / r_{i t a}$ is the present dimensionless turnaround radius. [Equation (19) corresponds to equation (4.7) in Bertschinger (1985b).] Substituting $r$ for $x$ [equation (12)] one can obtain the density in terms of dimensional physical parameters. The parameters of the self-similar solution $\left[\xi_{k}, \lambda\left(\xi_{k}\right)\right.$ and $\left.\lambda^{\prime \prime}\left(\xi_{k}\right)\right]$ that determine the density in the vicinity of every caustic must be obtained from numerical integration of equation (4) and equation (6) [or (4) and the approximate equation (7)].

\subsection{Medium with thermal velocity dispersion}

In this section, we derive semi-analytic expressions for the density profile in the vicinity of the caustics for non-zero velocity dispersion. Although in rigorous mathematical terms caustics, defined as manifolds of infinite density, would not form in the presence of a finite velocity dispersion, the density at the caustic "positions" would still be extremely high if the velocity dispersion is very small, as is the case for most dark matter candidates, and hence we still refer to these sharply-dense structures as caustics.

The motion of a medium with small thermal velocity dispersion can be approximated as a simultaneous evolution of many streams with different initial velocities $v$ at $\tau=\tau_{\text {ta }}$. The formation of the caustic in every stream occurs at different radii $x_{v}$. We denote the distance from the caustic as $\delta x_{v}=x-x_{v}$. We assume a linear relation between the relative position of the caustic and the initial velocity of the stream $v$

$x_{v}-x_{0}=\alpha_{k} v$,

where $v$ is the dimensionless velocity related to physical velocity $u$ as $u=\left(r_{i t a} / t_{i t a}\right) v$ and $\alpha_{k}$ is a negative constant to be determined numerically for every caustic. We will express the density as a function of the distance $\Delta x=x-x_{0}$ from the caustic in the stream with zero initial velocity

$\delta x_{v}=\Delta x-\alpha_{k} v$.

The major effect to consider in determining the maximum density in the 'caustic' in the medium with small thermal velocities is the shift of the position of the caustic in every stream with respect to the stream with $v=0$. As a result the $1 / \sqrt{-\Delta x}$ factor in equation (19) must be modified as in the following integral

$\rho(\Delta x)=\int \frac{f(v) d v}{\sqrt{-\Delta x+\alpha_{k} v}}$,

where $f(v)$ is the velocity distribution function at the turn around radius $q$ and the turn around time $\tau_{\text {ta }}$ corresponding to a chosen caustic and $f(v)$ is normalised to unity: $\int f(v) d v=1$. The above integral is simply the sum of densities in all streams at a distance $\Delta x$ from the true caustic in cold medium.

In the simplest case of the one-dimensional top-hat $(\mathrm{TH})$ velocity distribution 
$f_{T H}(v)= \begin{cases}\frac{1}{2 \sigma_{v}}, & \text { if }|v|<\sigma_{v} \\ 0 & \text { otherwise }\end{cases}$

we obtain

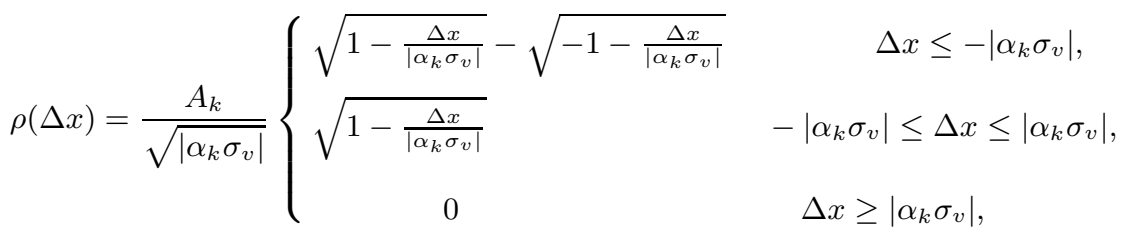

for the density profile near caustics, where $A_{k}$ is given by equation (20). One recovers equation (19) from equation (25) in the limit of $\sigma_{v}=0$.

In order to find the constant $\alpha_{k}$ we numerically solve the equation for $\lambda(\xi)$ in the presence of the initial nondimensional velocity perturbation $\delta \lambda_{0}^{\prime}$ in the initial conditions at $\xi=0$ so that

$\lambda(0)=1, \quad \lambda^{\prime}(0)=-\frac{8}{9}+\delta \lambda_{0}^{\prime}$.

The change of the maximum of $\lambda\left(\xi_{k}\right)=\max =\lambda_{k} \rightarrow \lambda_{k}+\delta \lambda_{k}$ in this case is found to be well approximated by a linear expression

$\delta \lambda_{k}=\Lambda_{k} \delta \lambda_{0}^{\prime}$,

where the coefficient $\Lambda_{k}$ depends on the caustic $k$ and shall be determined numerically. Differentiating equation (13) one finds the relation

$\frac{d x}{d \tau}=\tau^{-1 / 9}\left[\frac{8}{9} \lambda(\xi)+\lambda^{\prime}(\xi)\right]$,

between the dimensionless velocity $d x / d \tau$ and nondimensional functions. Therefore at the turnaround time, $\tau_{\text {ta }}$, the dimensionless velocity, $v$, given by equation $(21)$, is

$v=\tau_{\mathrm{ta}}^{-1 / 9} \delta \lambda_{0}^{\prime}$.

Taking the variation of equation (13) at $\tau_{k}$ and using equation (27) one obtains the distance between the caustic in the stream with velocity $v$ and the caustic in the stream with $v=0$

$x_{v}-x_{0}=\tau_{k}^{8 / 9} \Lambda_{k} \delta \lambda_{0}^{\prime}$.

Finally, recalling that $x_{v}-x_{0}=\alpha_{k} v$ [equation (21)] one obtains the expression for $\alpha_{k}$ in terms of $\Lambda_{k}$

$\alpha_{k}=\tau_{\text {ta }} \exp \left(\frac{8}{9} \xi_{k}\right) \Lambda_{k}$,

noting that $\Lambda_{k}<0$ and $\alpha_{k}<0$. Combining equations (20),(25) and (31) gives the full expression for the density in the vicinity of caustics for the top-hat velocity distribution function. We derive the density profile in the vicinity of a caustic for the exponential and Gaussian velocity distribution functions in Appendix B.

A much simpler and approximate expression for the density can be written as

$\rho(\Delta x)=A_{k} \begin{cases}(-\Delta x)^{-1 / 2} & \text { for } \quad \Delta \mathrm{x} \leq \Delta \mathrm{x}_{\mathrm{c}}, \\ \left(-\Delta x_{c}\right)^{-1 / 2} & \text { for } \Delta \mathrm{x}_{\mathrm{c}} \leq \Delta \mathrm{x} \leq 0, \\ 0 & \text { for } \Delta \mathrm{x}>0 .\end{cases}$

where

$\Delta x_{c}=\alpha_{k} \sigma_{v}=\sigma_{v} \tau_{\text {ta }} \exp \left(\frac{8}{9} \xi_{k}\right) \Lambda_{k}$,

note that $\Delta x_{c}$ is negative. The above expression approximates the maximum density for the exponential and Gaussian velocity distribution functions quite well (see Fig. 1). Physically this approximation means that the highest density in caustics is reduced by thermal velocities but since the amount of mass having the highest densities is small $\propto\left(-\Delta x_{c}\right)^{1 / 2} \propto \sigma_{v}^{1 / 2}$ it does not significantly affect the rest of the density distribution.

In order to evaluate the density profile in the vicinity of a caustic we numerically solve equation (4) with the mass distribution approximated by equation (7) and the initial conditions given by equation (26). We summarise the caustic parameters (including $\Lambda_{k}$ ) in Table 1 . 


\begin{tabular}{|c|c|c|c|c|}
\hline$k$ & $\xi_{k}$ & $\lambda_{k}=\lambda\left(\xi_{k}\right)$ & $\lambda_{k}^{\prime \prime}=\lambda^{\prime \prime}\left(\xi_{k}\right)$ & $\Lambda_{k}$ \\
\hline \hline 1 & 0.985 & 0.368 & -5.68 & -0.0704 \\
2 & 1.46 & 0.237 & -11.2 & -0.0254 \\
3 & 1.76 & 0.179 & -16.7 & -0.0135 \\
4 & 1.98 & 0.146 & -22.3 & -0.00854 \\
5 & 2.16 & 0.124 & -28.0 & -0.00591 \\
6 & 2.31 & 0.108 & -33.9 & -0.00437 \\
7 & 2.43 & 0.0959 & -39.8 & -0.00337 \\
8 & 2.55 & 0.0865 & -45.7 & -0.00266 \\
9 & 2.64 & 0.0790 & -51.7 & -0.00221 \\
10 & 2.73 & 0.0727 & -58.8 & -0.00182 \\
\hline
\end{tabular}

Table 1. In this table, we gives the nondimensional parameters of the first ten caustics obtained from the numerical fits made to the curves in the phase space (similar to those shown in Fig.A3.)

Rearranging (32), we arrive at the following full expression for the maximum density (which occurs at $\Delta x=\Delta x_{c}$ )

$\rho_{\max }=\frac{\sqrt{2} \pi^{2}}{8} \frac{e^{-11 \xi_{k} / 18}}{\sqrt{\lambda_{k}^{\prime \prime} \Lambda_{k}}} \frac{1}{\lambda_{k}^{2}}\left(\frac{t}{t_{\mathrm{ita}}}\right)^{-1 / 18} \frac{\rho_{H}}{\sqrt{\sigma_{v}}}$,

where $t$ is the present time. Factor $\left(t / t_{\text {ita }}\right)^{-1 / 18} \sigma_{v}^{-1 / 2}$ can be expressed in terms of radial component of the physical thermal velocity dispersion, $\sigma_{\mathrm{ph}}$, at the turnaround radius $R_{t a}$ at the turnaround time $t_{\mathrm{ta}}$. First, we note that $\sigma_{v}=\sigma_{\mathrm{ph}}\left(r_{\text {ita }} / t_{\text {ita }}\right)$, and then we use equation (3) to obtain $r_{\text {ita }} / t_{\text {ita }}=\left(r_{\text {ta }} / t\right)\left(t / t_{\text {ita }}\right)^{1 / 9}$. Combining both factors one obtains

$\left(\frac{t}{t_{\text {ita }}}\right)^{-1 / 18} \sigma_{v}^{-1 / 2}=\sigma_{\mathrm{ph}}^{-1 / 2}\left(\frac{r_{\mathrm{ta}}}{t}\right)^{1 / 2}$

We wish to stress that both $t$ and $r_{\mathrm{ta}}$ are present time and present turnaround radius while $\sigma_{\mathrm{ph}}$ is the physical velocity dispersion at the turnaround radius at the turnaround time. The thermal velocity dispersion at turnaround radius at the turnaround time can be estimated from the conservation of the phase space volume. The density at the turnaround radius is $D(1)=(3 \pi / 8)^{2} \approx 1.39$ times greater than $\rho_{H}\left(t_{\mathrm{ta}}\right)$ therefore $\sigma_{\mathrm{ph}}$ approximately $D(1)^{1 / 3} \approx 1.24$ times greater then the thermal velocity dispersion in the homogeneous universe at that time.

Thus, the maximum density only depends on the caustic, the velocity dispersion and the background Einstein-de Sitter density, $\rho_{H}$. The ratio of the maximum density to the background (Einstein-de Sitter density which should not be confused with the local halo density) is thus almost independent of any physical parameters. Using caustic parameters given in the previous table, we can evaluate the maximum density at caustic positions. In Table 2 , we summarise the value of $\rho / \rho_{H} / \sqrt{\sigma_{v}}$ and also of the local halo density for the first ten caustics.

\begin{tabular}{|c|c|c|c|c|c|c|c|c|c|c|}
\hline$k$ & 1 & 2 & 3 & 4 & 5 & 6 & 7 & 8 & 9 & 10 \\
\hline$\rho_{\max } / \rho_{H} / \sqrt{\sigma}_{v}$ & 11. & 24. & 39. & 56. & 74. & 95. & 117. & 139. & 165. & 190. \\
\hline$\rho_{\text {halo }} / \rho_{H}$ & 12. & 40. & 83. & 139. & 210. & 297. & 397. & 506. & 641. & 777. \\
\hline
\end{tabular}

Table 2. This table gives the maximum caustic density evaluated using (32) at the first ten caustic and also the halo density evaluated using the approximate expression (7) at the position of the caustics. Both of these densities are given as a ratio to the background Einstein de-Sitter density, $\rho_{H}$. The non-dimensional velocity dispersion, $\sigma_{v}$, is given by expression (35).

Thus, we can evaluate for a given velocity dispersion, the positions when the maximum caustic density becomes equivalent to the background density. Clearly for small values of $\sigma_{v}$ this would occur only at small radii for inner caustics and vice versa. The values of velocity dispersion for cold dark matter is very small and would be expected to be much smaller than unity. Thus, it is clear from Table 2 that the enhancement factor can be extremely high, for low values of the velocity dispersion $\sigma_{v}$.

\section{EMISSION MEASURE $(\mathcal{E} \mathcal{M})$ FROM DARK MATTER PARTICLE ANNIHILATION IN THE CAUSTICS}

The annihilation flux (in photons $/ \mathrm{cm}^{2} / \mathrm{s}$ ) can be written as

$\Phi_{\gamma}(\psi)=\frac{N_{\gamma}\langle\sigma v\rangle}{4 \pi m_{\chi}^{2}} \times \frac{1}{\Delta \Omega} \int_{\Delta \Omega} d \Omega \times(\mathcal{E M})$

where the emission measure, 


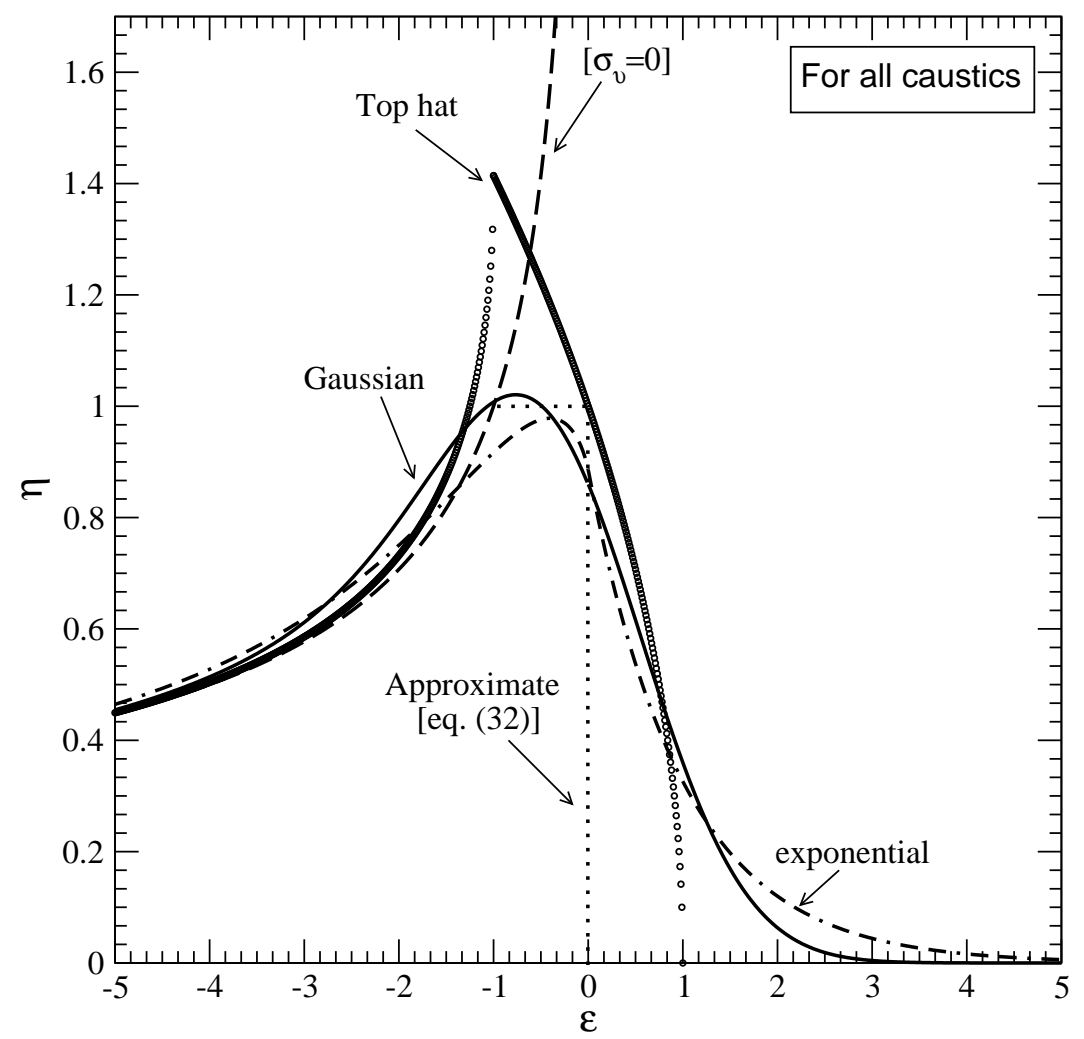

Figure 1. The rescaled density $\eta=\rho A_{k}^{-1}\left(\alpha_{k} \sigma_{v}\right)^{1 / 2}$ versus the rescaled distance $\epsilon=\Delta x \alpha_{k}^{-1} \sigma_{v}^{-1}$ from the caustic. Dashed line shows the case of cold matter $\left(\sigma_{v}=0\right)$, circles show the top-hat velocity distribution. Dashed-dotted and solid lines show the cases of the exponential and the Gaussian initial velocity distributions respectively. The dotted curve is a simple approximation given by equation (32), which is used in the evaluation of the emission measure in Section 4 and used also in Figure 2 . The density profile is evidently universal: it is independent of halo parameters and is valid for all of the caustics.

$\mathcal{E M}=\int_{\text {line of sight }} \rho^{2}(s) d s$,

is found by integrating the square of the density along the line of sight and over the solid angle $\Delta \Omega, m_{\gamma}$ is the mass of the candidate particle (e.g. neutralino), $N_{\gamma}$ is the number of photons produced per annihilation. To compute the first part of integral $N_{\gamma}\langle\sigma v\rangle /\left(4 \pi m_{\chi}^{2}\right)$ a supersymmetric model needs be selected. We shall not discuss this aspect here which is already extensively discussed in the relevant literatures (e.g. see Jungman, Kamionkowski and Griest 1996 and references therein). In this article, we obtain analytic expression for the emission measure (37), using our approximate expression for the density (32).

Here we are interested in calculating the $(\mathcal{E M})$ from the regions close to the caustic surfaces formed in cold matter. Clearly, the $(\mathcal{E M})$ is considerably higher when the line of sight is close to the tangent to the caustic surface. We estimate the $(\mathcal{E} \mathcal{M})$ in a small vicinity of this tangent. Although, in principle in order to obtain the emission measure (37) one can integrate expressions (B6) or (B7) or (B8) numerically, here we use our simple approximation (32) for the density profile in the vicinity of the caustic and make analytic estimates for the boost factor (37).

We assume that the density in the vicinity of the caustic can be approximated by equation (32). Figure 2 illustrates the geometry of the system. The figure shows the plane passing through the observer, $O$, the centre of the galaxy, $C$, and point $D$ where the line of sight $O P$ is tangential to the caustic sphere. The external and internal caustic spheres have the radius $R_{\mathrm{ex}}$ and $R_{\mathrm{in}}=R_{\mathrm{ex}}+\Delta x_{c}$ respectively (note $\Delta x_{c}$ is negative, see equation (33 and also Fig. 2). The density in the shell between two spheres is constant $\rho=A_{k}\left(-\Delta x_{c}\right)^{-1 / 2}$, while inside it falls as $\rho=A_{k}(-\Delta x)^{-1 / 2}$ where $\Delta x$ is the radial coordinate measured from point $D$ on the external sphere $(\Delta x<0)$.

We evaluate the $(\mathcal{E M})$ as a function of the angle $\theta$ measured from the line $O P$ upwards. First, we calculate the contribution to the emission measure along the line of sight which runs inside the angle POQ. In this case, no integral needs to be evaluated since the density is constant and the integral of density along the line of sight is the density times the length of the chord between two points where the line of sight crosses the external circle (e.g. $t T$ ).

The contribution to the emission measure, (37), from the lines crossing inside POQ is 


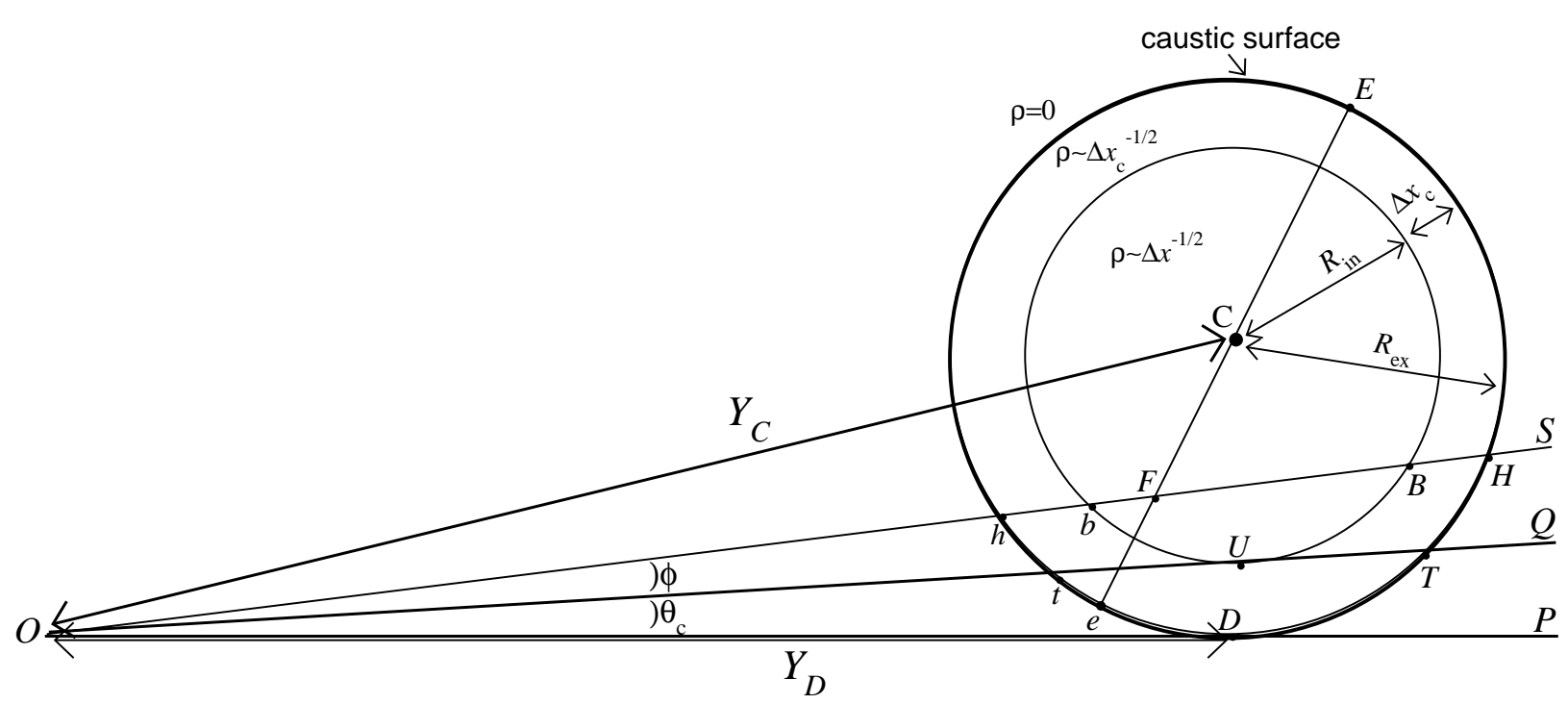

Figure 2. An approximation is used in which the caustic is the outer-shell (larger circle) outside which the density is zero, inside a layer of thickness $\Delta x_{c}$ the density is constant taken to be $\rho_{c}=A_{k} \Delta x_{c}^{-1 / 2}$. In the interior of this shell the density falls as $1 / \sqrt{-\Delta x}$ where $\Delta x$ is the radial coordinate measured from the tangent point $D(\Delta x<0)$.

$\mathcal{E} \mathcal{M}(\theta)=\int \rho^{2} d s=\rho_{c}^{2} L_{c h}(\theta)$

where $L_{c h}(\theta)$ is the length of the chord. The equation of the circle in the vicinity of the tangential point $D$ is to the lowest order

$\Delta x=-\frac{y^{2}}{2 R_{\mathrm{ex}}} \quad$ or $\quad y= \pm \sqrt{-2 R_{\mathrm{ex}} \Delta x}$

where $y$ is the coordinate along DP measured from point D. For example, the length of the chord $t T$ is

$L_{t T}=\sqrt{\left(y_{T}+y_{t}\right)^{2}+\left(\Delta x_{T}-\Delta x_{t}\right)^{2}} \approx y_{T}+y_{t}$,

because $\Delta x$ is of the higher order in $y$ and thus the second term can be neglected $\left(y_{T}>0\right.$ and $\left.y_{t}<0\right)$. Neglecting the difference between $\Delta x_{t}$ and $\Delta x_{T}$ allows to approximate them as $\Delta x_{t} \approx \Delta x_{T} \approx-L_{O D} \theta=-Y_{D} \theta$ (where $Y_{D}=\sqrt{Y_{C}^{2}-R_{\mathrm{ex}}^{2}}$ is the distance from the observer to the caustic and $Y_{C}$ is the distance from the observer $\mathrm{O}$ to the centre $\mathrm{C}$ ) assuming $\theta$ is small. Combining this approximation with the second equation (39) the length of the chord can be written in a simple form

$L_{c h}=2 \sqrt{2 R_{\mathrm{ex}} Y_{D}} \sqrt{\theta}$,

and thus,

$\mathcal{E M}(\theta)=2 \rho_{c}^{2} \sqrt{2 R_{\mathrm{ex}} Y_{D}} \sqrt{\theta} \quad$ for $\quad \theta<\theta_{c}=\angle P O Q \approx \frac{-\Delta x_{c}}{Y_{D}}$.

Next, we suppose that the line of sight is along OS in which case it crosses the region where the density falls as $(-\Delta x)^{-1 / 2}$. The integral (37) is now the sum of three parts:

$\mathcal{E} \mathcal{M}_{h H}=(\mathcal{E M})_{h b}+(\mathcal{E M})_{B H}+(\mathcal{E} \mathcal{M})_{b B}=(\mathcal{E M})_{b B}+\rho_{c}^{2}\left(L_{h b}+L_{B H}\right)$.

The length $L_{h b}+L_{B H}=L_{h H}-L_{b B}$ can be easily evaluated in a similar manner as before. The length of the chord $h H$ is given by equation (41) while for the chord $b B$ one needs to substitute $R_{\text {in }}=R_{\mathrm{ex}}+\Delta x_{c}$ for $R_{\mathrm{ex}}, \sqrt{Y_{C}^{2}-R_{\text {in }}^{2}}$ for $Y_{D}$, and the angle $\phi=\angle Q O S$ for $\theta$. For small angles $\theta$ and small $\Delta x_{c}$ the change in the radius and distance to the caustic result in the higher order corrections and can be neglected, yielding

$L_{b B}=2 \sqrt{2\left(R_{\mathrm{ex}}+\Delta x_{c}\right) \sqrt{Y_{C}^{2}-R_{\mathrm{in}}^{2}}} \sqrt{\phi} \approx 2 \sqrt{2 R_{\mathrm{ex}} Y_{D}} \sqrt{\theta-\theta_{c}}$.

where $\theta_{c}=-\Delta x_{c} / Y_{D}=A_{k}^{2} /\left(\rho_{c}^{2} Y_{D}\right)$ and all distances are given in units of equation (13). Thus, the $(\mathcal{E} \mathcal{M})$ from the parts of the line of sight with constant density becomes

$(\mathcal{E M})_{h b}+(\mathcal{E M})_{B H}=2 \rho_{c}^{2} \sqrt{2 R_{\mathrm{ex}} Y_{D}}\left(\sqrt{\theta}-\sqrt{\theta-\theta_{c}}\right) \quad$ for $\quad \theta>\theta_{c}=-\frac{\Delta x_{c}}{Y_{D}}$. 
The complicated part of contribution to the integral (37) comes from the line of sight $b B$. Here, we actually need to calculate the integral (37) where the density is no longer constant but falls as $(-\Delta x)^{-1 / 2}$. First of all we need to express the distance $|\Delta x|=L_{e F}$ in terms of the line of sight distance $z=L_{h F}$. This can be easily done by solving the intersecting chords relation $L_{h F} \times L_{F H}=L_{e F} \times L_{F E}$ for $\Delta x\left(L_{F E}=2 R_{\mathrm{ex}}-|\Delta x|\right.$ and $L_{F H}=L-z$ (where $L=L_{h H}$ ) which yield

$(\mathcal{E M})_{b B}=\rho_{c}^{2} \int_{z_{b}}^{z_{B}} \rho(z)^{2} d z=\rho_{c}^{2} \int_{z_{b}}^{z_{B}} \frac{d z}{R_{\mathrm{ex}}-\sqrt{R_{\mathrm{ex}}^{2}-L_{b B} z+z^{2}}}$

where $z_{b}$ and $z_{B}$ correspond to points $b$ and $B$ respectively. The integral (46) can be written in close form

$I=\frac{R_{\mathrm{ex}}}{L}\left\{\operatorname{arctanh}\left[\frac{2 R_{\mathrm{ex}} L(L-2 z) \sqrt{R_{\mathrm{ex}}^{2}-L z+z^{2}}}{\left.z(L-z)\left(4 R_{\mathrm{ex}}^{2}+L^{2}\right)-2 R_{\mathrm{ex}}^{2} L^{2}\right)}\right]-\ln \left(\frac{L-z}{z}\right)\right\}-\ln \left(2 \sqrt{R_{\mathrm{ex}}^{2}-L z+z^{2}}-L+2 z\right)$

The limits in the integral are to linear order

$z_{b}=\sqrt{2 R_{\mathrm{ex}} Y_{D}}\left(\sqrt{\theta}-\sqrt{\theta-\theta_{c}}\right) \quad$ and $\quad z_{B}=\sqrt{2 R_{\mathrm{ex}} Y_{D}}\left(\sqrt{\theta}+\sqrt{\theta-\theta_{c}}\right)$.

Substituting the limits into equation (47) is straightforward but results can be lengthy and complex. In addition, despite the exact form of the integral (47) we use the accurate limits only to the lowest order. Thus, we simplify the equation for $(\mathcal{E M})$ by taking the lowest order terms in the series expansion for small $\theta$ and $\theta_{c}$.

$(\mathcal{E M})(\theta)=\rho_{c}^{2} \sqrt{\frac{2 R_{\mathrm{ex}}}{Y_{D}}} \frac{1}{\sqrt{\theta}} \ln \left(\frac{\sqrt{\theta}+\sqrt{\theta-\theta_{c}}}{\sqrt{\theta}-\sqrt{\theta-\theta_{c}}}\right)$.

Thus, collecting various expressions for the emission measure together, we arrive at

$\mathcal{E M}=\rho_{c}^{2}\left\{\begin{aligned} 0 & \text { for } \quad \theta<0, \\ 2 \sqrt{2 R_{\mathrm{ex}} Y_{D}}\left(\sqrt{\theta}-\sqrt{\theta-\theta_{c}}\right)+\sqrt{2 R_{\mathrm{ex}} / Y_{D}} \theta^{-1 / 2} \ln \left(\frac{\sqrt{\theta}+\sqrt{\theta-\theta_{c}}}{\sqrt{\theta}-\sqrt{\theta-\theta_{c}}}\right) & \text { for } \quad \theta>\theta_{c} .\end{aligned}\right.$

where once again we mention that $R_{\text {ex }}$ is the radius of the caustic sphere, $Y_{D}$ is the distance to the caustic surface, $\theta_{c}=-\Delta x_{c} / Y_{D}=A_{k}^{2} /\left(\rho_{c}^{2} Y_{D}\right)$ and all distances are given in units of equation (13).

\section{$5 \quad$ EMISSION FROM M31}

Detailed application of our results to haloes especially minihaloes (Diemand, Moore \& Stadel 2005) shall be presented in a forthcoming work. Here, as an exercise, we apply the results of the previous sections to a very simple model of M31. Emission from M31 dark matter halo has already been studied, largely in the context of dark matter search experiment CELEST (e.g. see Nuss et al. 2002 and Falvard et al. 2004). M31 is situated at a distance of 780 Kpc from the centre of MilkyWay (MW) with a present turnaround radius which is taken to be at about $800 \mathrm{Kpc}$ (Sandage 1986, Karachentsev 2002). In fact M31 and MW can be considered to be embedded in a common halo, however here in our very simple model we consider M31 to have its own halo. The first caustic, which is the closest to us cuts the radius joining the centre of MW to the centre of M31 at a distance of about $500 \mathrm{Kpc}$ from us. The tangent point to this caustic lies at about $700 \mathrm{Kpc}$ from the centre of MW. This caustic has a thickness of about $0.115 \mathrm{Kpc}$ and subtends an angle $\left(\theta_{c}\right)$ of about $0.006^{\circ}$ at the centre of Milky Way. The maximum emission measure from this caustic, using the expressions (50) for a velocity dispersion of $\sigma_{v}=0.001$ is given in Table 3 . We assume a field of view of $1^{o}$, which is at the lowest-end for most detectors.

\begin{tabular}{|c|c|c|c|c|c|c|c|}
\hline$k$ & $r(\mathrm{kpc})$ & $\delta r(\mathrm{kpc})$ & $\theta_{c}^{o}$ & $\rho_{\max }$ & $\int_{0}^{\theta_{c}} \mathcal{E} \mathcal{M} d \theta$ & $\int_{\theta_{c}}^{1^{\circ}} \mathcal{E} \mathcal{M} d \theta$ & $\operatorname{Total}(\mathcal{E} \mathcal{M}) \mathrm{Gev}^{2} \mathrm{~cm}^{-5} \mathrm{c}^{-4}$ \\
\hline 1 & 300 & 0.115 & 0.006 & $350 \rho_{H}$ & $260 \rho_{H}^{2} r_{\mathrm{ta}}$ & $5 \times 10^{5} \rho_{H}^{2} r_{\mathrm{ta}}$ & $4 \times 10^{24}$ \\
\hline
\end{tabular}

Table 3. This table gives approximate values for various caustic parameters and also the emission measure for the first outer caustic of M31, which is the nearest to Milky Way. We take the non-dimensional velocity dispersion to be $\sigma_{v} \sim 0.001$ which would give only a very modest estimate of the maximum density. For neutralino, this parameter is smaller by a few orders of magnitude leading to a significantly higher density maximum but at the same time smaller angle, $\theta_{c}$. Expression (35) can be used to transform between the physical velocity dispersion, $\sigma_{p h}$, and the non-dimensional velocity dispersion, $\sigma_{v}$.

The emission measure from the first caustic of M31 is at least of the same order ${ }^{2}$ as that for example from the

2 Different values for the emission measure form the galactic centre has been evaluated ranging from $10^{21}$ to $10^{31}$ depending on 


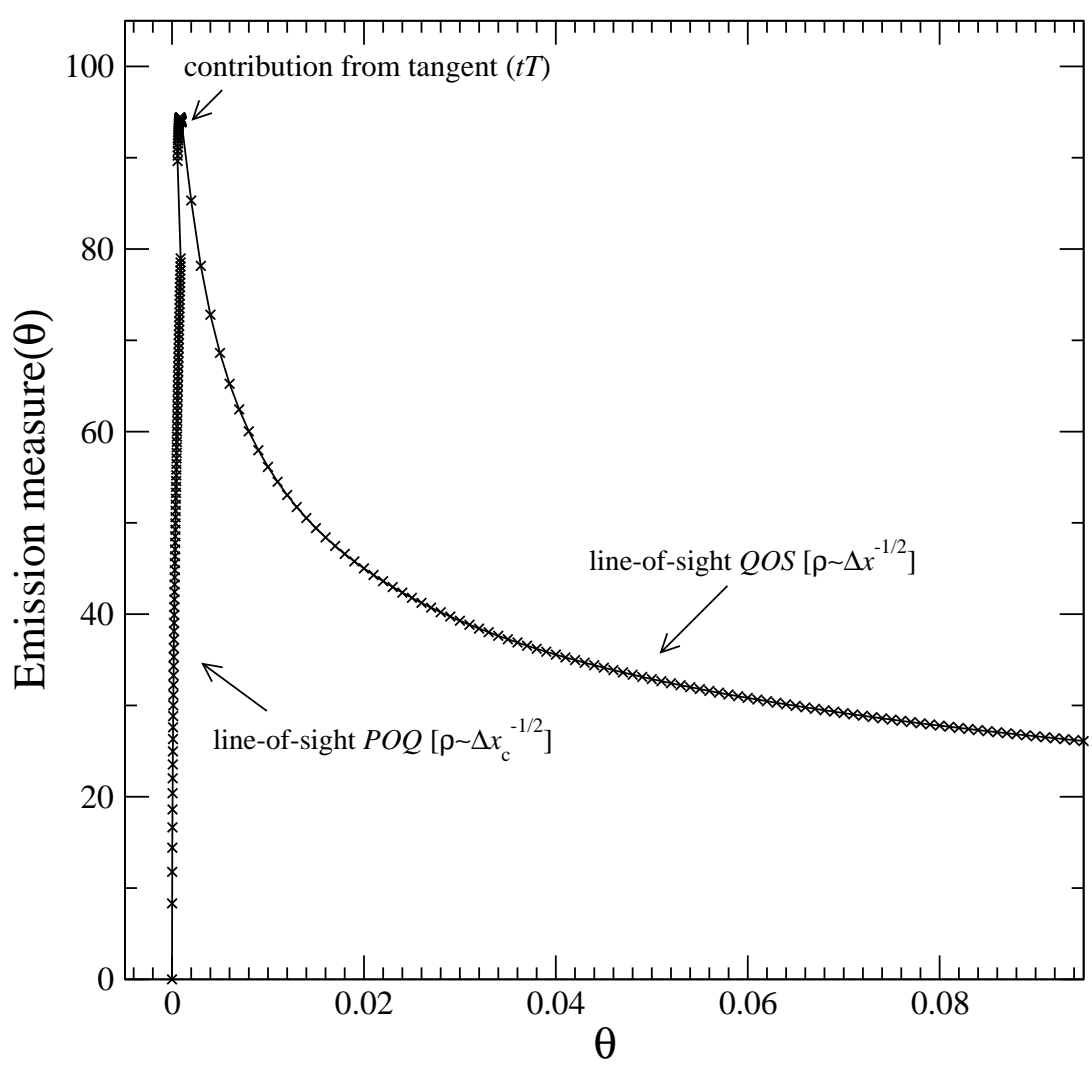

Figure 3. Contribution to the emission measure (37) along different lines of sights as marked on Fig. 2. All values are in non-dimensional coordinates.

centre of Milky Way. Here, we have used a moderate value of $\sigma_{v}=0.001$, a lower value would sharply increase the maximum density as is evident from expressions (32) and (36) although it would also reduce the thickness of the caustic. The simple exercise in this section also demonstrates that emission measure from caustics can serve as a mean to put bound on the mass of the dark matter particle candidates.

\section{DISCUSSION}

In this work, we have evaluated the density profile near caustics which arise in the selfsimilar scenario of the formation of dark matter haloes. We have obtained a universal semi-analytic expression for this density profile and its maximum value in the presence of an infinitesimal velocity dispersion. We have shown that the maximum density at the caustics depends primarily on the velocity dispersion and is far higher than the halo density at the position of the caustic. The radius at which the caustic density approaches the background halo density depends on the value of the velocity dispersion and is expected to arise only for the innermost caustics. We have then evaluated the emission measure from the caustics and applied it to the concrete example of M31 outermost caustic, which is closest to us. This example demonstrates that caustics can be promising sources for dark matter search experiments. Application of our results to other haloes, in particular to small haloes which have not had significant mergers, to specific search experiments such as HESS and also to direct detection experiments remains to be done.

Although we have considered an Einstein-de Sitter Universe, we expect our results to give reasonable approximation for the $\Lambda$ CDM Universe as well. The rôle of dark energy becomes significant at rather small redshifts $(\sim 0.2)$ which we expect to be well after the formation of the typical dark matter haloes we consider here. Furthermore, once a particle turns around and collapses, it separates from the background expansion and its subsequent motion should not be effected by the $\Lambda$ term. The second caveat in our consideration is the assumption of spherical symmetry of haloes and their cold accretion which does not hold in general cases. Finally, the third and probably most serious

various physical assumptions such as the presence or absence of a central core, a central cusp, or a central supermassive black hole (see for example Stoehr et al. 2004; Evans, Ferrer and Sarkar 2004). 
problem is associated with the smooth precollapse conditions on galactic scale that contradicts the hierarchical clustering scenario. The cold dark matter models predict a relatively high level of small scale perturbations that result in the formation of small gravitationally bound haloes that are assembled into more massive haloes at later times. Thus, the dark matter accrets onto halos of galactic size in the form of smaller haloes that may significantly affect the density in the vicinity of caustics. However, there is a possibility that in cold dark matter models the smallest haloes can survive the tidal destruction in more massive halos (Diemand, Moore \& Stadel 2005). In this case our results can be applied directly. This issue will be studied in detail in the following work.

We believe that the current results represent a step toward building a more comprehensive theoretical model of the gravitational collapse. A more elaborate study of caustic distributions and density profiles, in broader settings without the assumptions of spherical symmetry, inertial trajectories or smooth initial conditions remains a challenging task.

\section{ACKNOWLEDGEMENTS}

We are grateful to Joe Silk for many useful comments and ongoing collaboration and to Stephane Colombi, Pierre Salati and Brent Tully for discussions. Special thanks go to Jacques Colin and Uriel Frisch for invaluable supports at the Observatoire de la Côte d'Azur where major part of this work was carried out. RM was supported by a Marie Curie HPMF-CT 2002-01532 and a European Gravitational Observatory (EGO) fellowship at the school of astronomy of the university of Cardiff, UK.

\section{REFERENCES}

Alard C., Colombi S., A cloudy Vlasov solution, astro-ph/0406617

Arnold V.I., Shandarin S., Zel'dovich Ya.-B, 1982, Geophysical and Astrophysical Fluid Dynamics, 20, 111

Arnold V.I., 1986, Catastrophe Theory, Springer-Verlag Telos, 2nd Edition

Arnold V.I., 1990, Singularities of caustics and wave fronts, Kluwer Academic publishers, Mathematics and its applications (Soviet Series) volume 62.

Bergström L., Edsjö, Gunnarsson C. 2001, Phys. Rev D 63, 083515

Bertschinger E. 1985a, ApJ 58, 1

Bertschinger E. 1985b, ApJ 58, 39

Bharadwaj S.,Sahni V., Sathyaprakash B.S., Shandarin S.F., Yess C., 2000, ApJ 528, 21

Binney J., 2004, MNRAS 350, 939

Diemand J., Moore B., Stadel J., 2005, Nature 433, 389

Dubinski J., Carlberg R., 1991, ApJ 378, 496

Evans N.W., Ferrer F., Sarkar S., 2004, Phys. Rev. D 69, 123501

Falvard A. Giraud E., Jacholkowska A., Lavalle J., Nuss E., Piron F., Sapinski M., Salati P., Taillet R., Jedamzik K., Moultaka G. 2004, Atropart. Phys. 20, 467

Fillmore J.A., Goldreich P. 1984, ApJ 281, 1

Gondolo P., Silk J., 1999, Phys. Rev. Lett. 83, 1719

Gott J.R., 1975, ApJ 201, 296

Gunn J.E. 1977, ApJ 218, 592

Henriksen R.N. 2004, MNRAS 355, 1217

Jungman G., Kamionkowski M., Griest K., 1996, Phys. Rep. 267, 195

Karachentsev I.D. et al., 2002, A\& A 389, 812

Klypin A., Kravtsov A.V., Valenzuela O., Prada F., 1999, ApJ 52, 82

McGaugh S.S, Barker M.K., de Blok W.J.G., 2003, ApJ 584, 566

Melott A.L., Shandarin S.F., Plinter R.J., Suto Y., 1997, ApJL 479, 79

Moore, B., Ghigna S., Governato F., Lake G., Quinn T., Stadel J., Tozzi P., 1999, ApJL 524, 19

Navarro J.F., Frenk C.S., White S.D.M., 1996, ApJ 62, 563

Navarro J.F., Frenk C.S., White S.D.M., 1997, ApJ 490, 493

Nuss E. et al. ,in Semaine de l'Astrophysique Francaise, Paris, France, June 24-29, 2002, Eds.: F. Combes and D. Barret, EdP-Sciences (Editions de Physique), 279

Roytvarf A., 1994, Physica D 73, 189

Sahni V., Shandarin, S., 1996, MNRAS 282, 641

Salati P., 2004, in 4ème semaine de l'astrophysique francaise, Paris, eds: F. Combes et al., EDP Sciences

Sandage A., 1986, ApJ 307, 1

Shandarin S.F., Zel'dovich Ya.B. 1989, Rev. Mod. Phys. 61, 185

Sikivie P., Tkachev I.I., Wang Y. 1997, Phys. Rev. D 56, 1863

Splinter R.J., Melott A.L., Shandarin S.F., Suto Y., 1998, ApJ 497, 38

Stoehr F., White S.D.M., Springel V., Tormen G., Yoshida N., 2003, MNRAS 345, 1313

Zel'dovich Ya.B., 1970, A\&A 5, 84 


\begin{tabular}{|r|c|c|c|}
\hline $\mathrm{k}$ & $\xi$ & $\lambda$ & $\mathrm{d}^{2} \lambda / \mathrm{d} \xi^{2}$ \\
\hline \hline 1 & $0.985(0.988)$ & $0.368(0.364)$ & $-5.86(-6.35)$ \\
\hline 2 & $1.46(1.46)$ & $0.237(0.236)$ & $-11.2(-11.6)$ \\
\hline 3 & $1.76(1.76)$ & $0.179(0.179)$ & $-16.7(-16.9)$ \\
\hline 4 & $1.98(1.98)$ & $0.146(0.145)$ & $-22.3(-22.4)$ \\
\hline 5 & $2.16(2.15)$ & $0.124(0.123)$ & $-28.0(-28.1)$ \\
\hline 6 & $2.31(2.30)$ & $0.108(0.108)$ & $-33.9(-33.8)$ \\
\hline 7 & $2.43(2.43)$ & $0.096(0.096)$ & $-39.8(-39.6)$ \\
\hline 8 & $2.55(2.54)$ & $0.087(0.086)$ & $-45.7(-45.6)$ \\
\hline 9 & $2.64(2.64)$ & $0.079(0.079)$ & $-51.7(-51.5)$ \\
\hline 10 & $2.73(2.73)$ & $0.073(0.073)$ & $-57.8(-57.6)$ \\
\hline
\end{tabular}

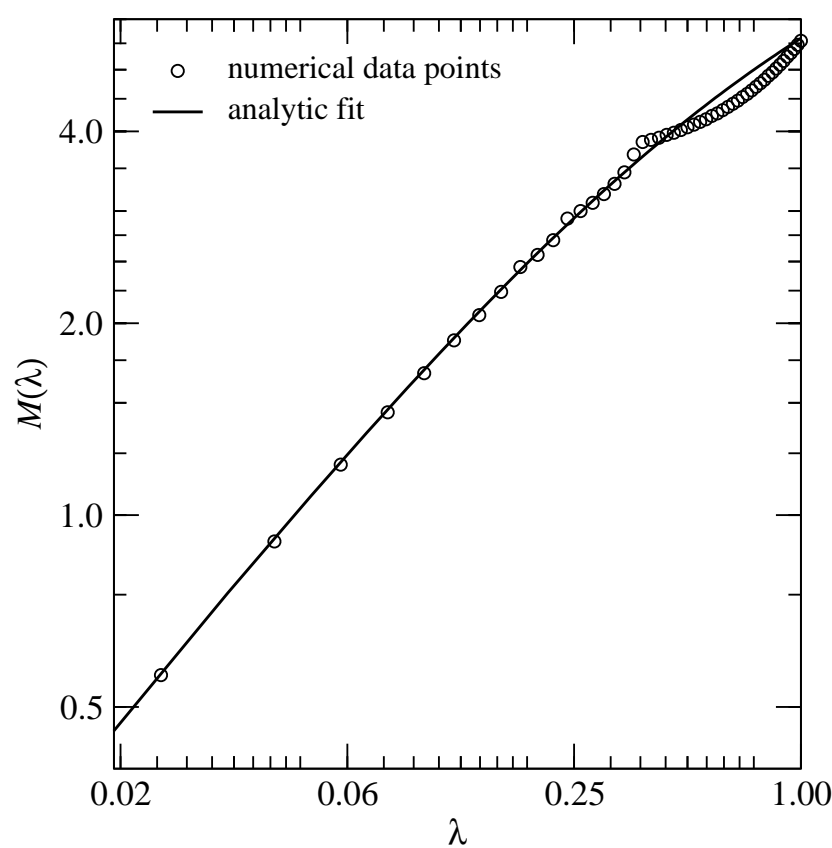

Figure A1. The fit (solid line) $M(\lambda)=11.2 \lambda^{3 \cdot / 4} \cdot /\left(1 .+\lambda^{0.75}\right)$ to the data points (open circles) taken from Table 4 of Bertschinger 1985b. The above table shows our values and Bertschinger 1985b values (given inside brackets) for $\lambda, \xi$ and $d^{2} \lambda / d \xi^{2}$ for the first ten caustics.

\section{APPENDIX A: NUMERICAL SIMULATIONS}

There is a computational difficulty in solving equation (4) which is singular at $\lambda=0$ where the velocity becomes infinite and changes discontinuously from negative to positive. In order to avoid this problem a small amount of angular momentum, of the form $J^{2} / \lambda^{3}$ is added the RHS of equation (4) where hereafter the value of $J=10^{-9}$ is adopted (Bertschinger 1985b).

Further computational issues arise in solving (4) and (6). The outline of the numerical procedure is as follows: a guess is first made for the mass distribution e.g., $M(\lambda)=M_{\mathrm{ta}} \lambda^{3 / 4}$ for $\lambda \leq 1$. Then equation (4) is integrated to obtain $\lambda(\xi)$. This is used to obtain a new approximation to $M(\lambda)$ and so on until a self-consistent solution is found (see Bertschinger 1985b for full details). In this article we have chosen the fitting formula (7) instead of (6), which as shown in Fig. A1 works well for small values of $\lambda$.

The solutions to equations (4) and (7) is plotted in Fig. A2 which can be viewed as the trajectory and phase diagram of one particle during the course of evolution of the halo, or as a snapshot of the positions of many particles in the halo. The change in real and phase space for finite velocity dispersion is clear: velocity dispersion leads to the broadening of caustics. The density at caustics no longer diverges but has a maximum cut-off determined by the velocity dispersion, which is the main result of this work.

The small velocity dispersion effects are primarily related to defocusing of trajectories of particles with different thermal velocities in the vicinity of caustics. Imagine the evolution of large number of streams each corresponding to a particular value of thermal velocity then these streams will produce a caustic at a slightly different radii. The resulting density field becomes the sum of densities in every stream. We assume that each stream evolves in the same gravitational field generated by the mass distribution of the cold medium [equation (7)].

We consider the process in the nondimensional coordinates $\xi, \lambda, \lambda^{\prime} d \lambda / d \xi$. As we have mentioned in Subsection 3.2 , the effects of thermal velocity dispersion can be considered by adding small velocities $\delta \lambda_{0}^{\prime}=\delta \lambda^{\prime}(0)$ to the initial velocity. Thus, the initial conditions become

$\lambda_{0} \equiv \lambda(0)=1, \quad \lambda_{0}^{\prime} \equiv \lambda^{\prime}(0)=-\frac{8}{9}+\delta \lambda_{0}^{\prime}$.

The result of the integration is shown in Fig. A3 for the first caustic. For small values of $\delta \lambda_{0}^{\prime}$, the major effect on the caustic is the change of the maximum value $\lambda_{k}$ that can be well-approximated by a linear function

$\delta \lambda_{k}=\Lambda_{k} \delta \lambda_{0}^{\prime}$

as is evident from Fig. A4. We find $\Lambda_{k}$ by fitting the numerical results for every caustic $k=1, \ldots, 10$. 

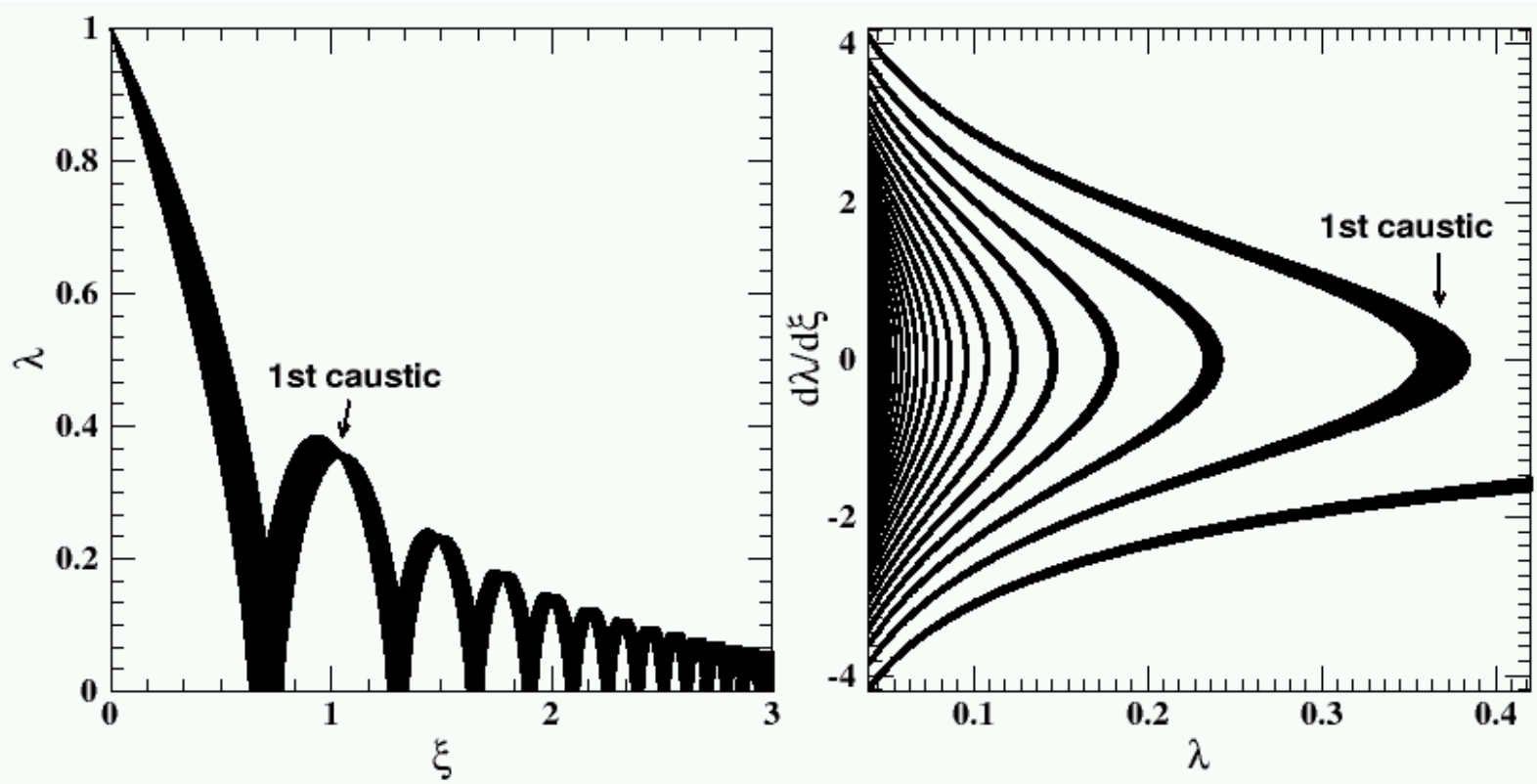

Figure A2. The left plot is the non-dimensional trajectory $\lambda(\tau)$ for a collisionless fluid, with minute velocity dispersion, given by similarity solution to equations (4) and (7). The particles reach their maximum radius at $\lambda=1$. The non-dimensional coordinate $\lambda$ and the non-dimensional time $\xi$ at the maxima depend almost linearly on the initial velocity of the particle. The right plot is a small portion of the phase diagram given by the similarity solution. Each particle travels along the entire curve and at a given time there is a particle at each point on the curve. As we go to smaller and smaller radii, the strips become narrower and more closely packed and finally resemble a smooth distribution.

\section{APPENDIX B: THE DENSITY PROFILES FOR THE TOP-HAT, EXPONENTIAL AND GAUSSIAN VELOCITY DISTRIBUTIONS}

We evaluated the density profile in the vicinity of a caustic for the top-hat initial thermal velocity distribution

$f_{T H}(v)=\rho_{0}\left\{\begin{array}{lr}\frac{1}{2 \sigma_{v}} & |v|<\sigma_{v} \\ 0 & \text { otherwise }\end{array}\right.$

in Sec. 3.2 [equation (25)]. Using similar approach one also can derive the density profiles for the exponential and Gaussian velocity distributions

$$
\begin{aligned}
& f_{E}(v)=\rho_{0} \frac{1}{2 \sigma_{v}} \exp \left(-\frac{|v|}{\sigma_{v}}\right) \\
& f_{G}(v)=\rho_{0} \frac{1}{\sqrt{2 \pi} \sigma_{v}} \exp \left(-\frac{v^{2}}{2 \sigma_{v}^{2}}\right) .
\end{aligned}
$$

Thus, deriving the density profile in the vicinity of a caustic in both cases consists in straightforward evaluations of a few integrals. However, one can simplify the calculations by introducing the scaled distance from the caustic $\epsilon$ and the scaled density $\eta$

$\epsilon=\frac{\Delta x}{\left|\alpha_{k} \sigma_{v}\right|}, \quad \eta=\frac{\rho}{A_{k}\left|\alpha_{k} \sigma_{v}\right|^{-1 / 2}}$

In terms of these variables the density of cold dark matter with zero velocity dispersion has a simple form

$\eta_{\sigma_{v}=0}= \begin{cases}(-\epsilon)^{-1 / 2} & \text { for } \epsilon<0 \\ 0 & \text { for } \epsilon>0\end{cases}$

and for the top-hat velocity distribution function, with non-vanishing $\sigma_{v}$, we have

$\eta_{\mathrm{TH}}= \begin{cases}\sqrt{1-\epsilon}-\sqrt{-1-\epsilon} & \text { for } \epsilon \leq-1, \\ \sqrt{1-\epsilon} & \text { for }-1 \leq \epsilon \leq 1, \\ 0 & \text { for } \epsilon \geq 1 .\end{cases}$ 


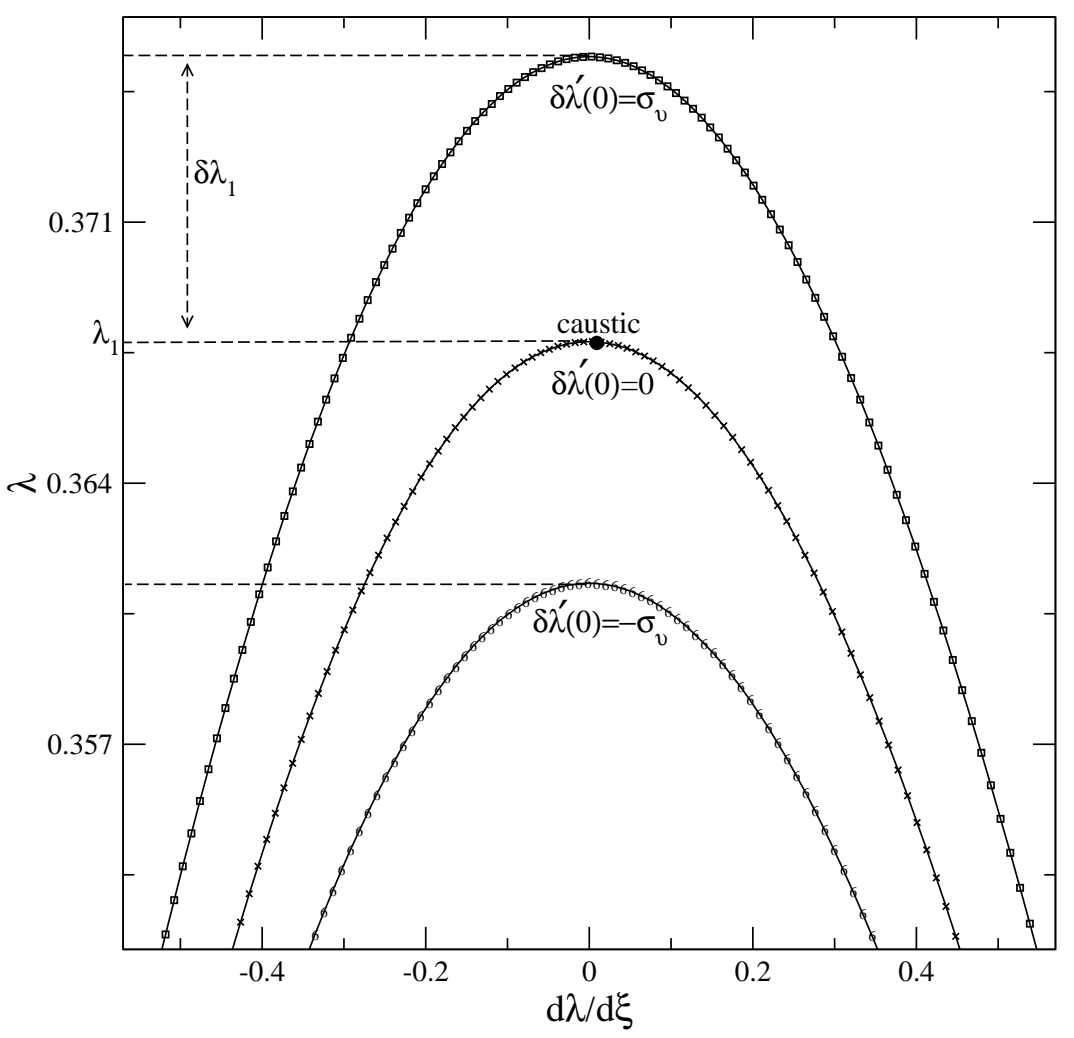

Figure A3. The flipped plot of the phase space around the first caustic for three values of the initial velocity perturbation, $\sim \delta \lambda_{0}$. Phase plots are used to evaluate the density at the caustics in the presence of a small velocity dispersion.
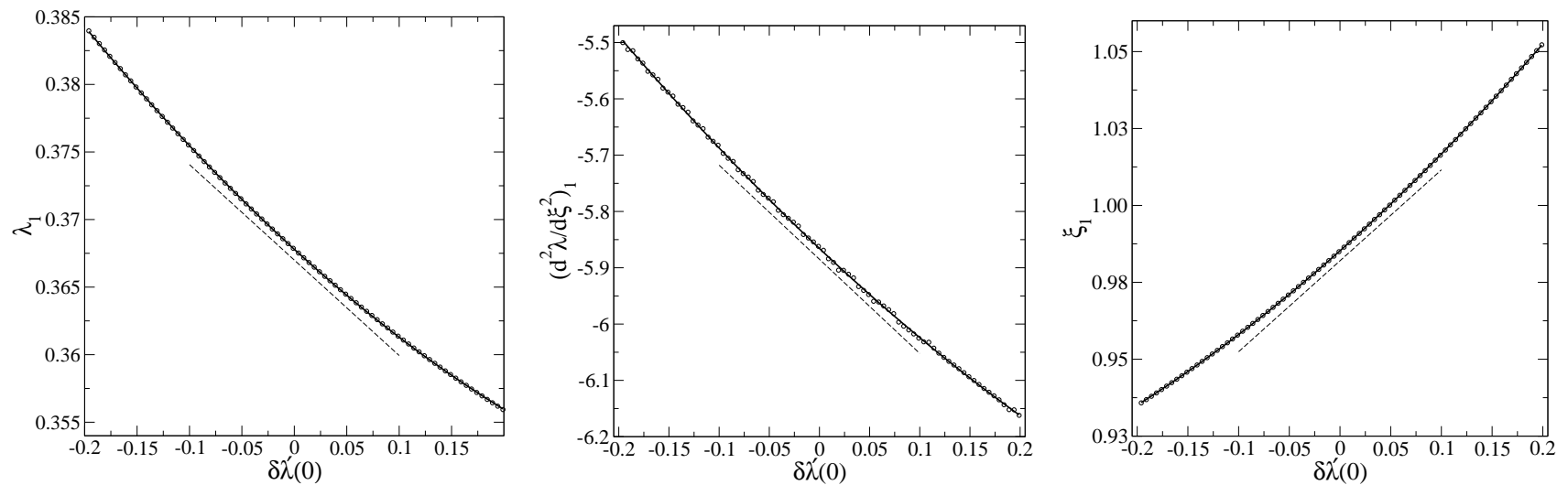

Figure A4. The left plot shows the variation of the non-dimensional position of the first caustic with the initial velocity perturbation, $\delta \lambda^{\prime}(0)$. The middle and right panels show the variations of the second derivative and the time of formation of the first caustic with velocity dispersion, respectively. The quadratic fits (solid line) are also shown. The dashed straight lines demonstrate that for very small velocity dispersion, a linear function would be equally appropriate. Similar fits were obtained for the first ten caustics. 
For the exponential velocity distribution function one obtains

$\eta_{\mathrm{E}}= \begin{cases}\frac{\sqrt{\pi}}{2}\left\{\mathrm{e}^{-\epsilon}[1-\operatorname{erf}(\sqrt{-\epsilon})]-\mathrm{i} \mathrm{e}^{\epsilon} \operatorname{erf}(\mathrm{i} \sqrt{-\epsilon})\right\} & \text { for } \quad \epsilon<0 \\ \frac{\sqrt{\pi}}{2} \mathrm{e}^{-\epsilon} & \text { for } \quad \epsilon>0\end{cases}$

and finally for the Gaussian velocity distribution function the density is as follows

$\eta_{\mathrm{G}}= \begin{cases}\sqrt{\left|\frac{\epsilon}{8 \pi}\right|} \mathrm{e}^{-\epsilon^{2} / 4}\left[2 \pi \operatorname{BesselI}\left(-\frac{1}{4}, \frac{\epsilon^{2}}{4}\right)-\sqrt{2} \operatorname{BesselK}\left(\frac{1}{4}, \frac{\epsilon^{2}}{4}\right)\right] & \text { for } \epsilon \leq 0 \\ \sqrt{\left|\frac{\epsilon}{4 \pi}\right|} \mathrm{e}^{-\epsilon^{2} / 4} \operatorname{BesselK}\left(\frac{1}{4}, \frac{\epsilon^{2}}{4}\right) & \text { for } \epsilon \geq 0 .\end{cases}$

The density profiles $\eta=\eta(\epsilon)$ are shown in Fig. 1 .

We also show a simple approximation used in further calculations

$\eta_{\mathrm{A}}= \begin{cases}(-\epsilon)^{-1 / 2} & \text { for } \epsilon \leq-1, \\ 1 & \text { for }-1 \leq \epsilon \leq 0, \\ 0 & \text { for } \epsilon>0 .\end{cases}$ 